\title{
Electromagnetic energy harvesting from vibrations of multiple frequencies
}

\author{
Bin Yang ${ }^{1}$, Chengkuo Lee ${ }^{1,2}$, Wenfeng Xiang ${ }^{2}$, Jin Xie ${ }^{1}$, Johnny Han He ${ }^{1}$, \\ Rama Krishna Kotlanka ${ }^{1}$, Siew Ping Low ${ }^{2,3}$ and Hanhua Feng ${ }^{1}$ \\ ${ }^{1}$ Institute of Microelectronics (IME), A*STAR (Agency for Science, Technology and Research), \\ 11 Science Park Road, Singapore Science Park II, Singapore 117685 \\ ${ }^{2}$ Department of Electrical \& Computer Engineering, National University of Singapore, 4 Engineering \\ Drive 3, Singapore 117576 \\ ${ }^{3}$ Department of Electrical and Electronics Engineering, Imperial College London, \\ South Kensington Campus, London SW7 2AZ, UK \\ E-mail: yangb@ime.a-star.edu.sg and elelc@nus.edu.sg
}

Received 1 October 2008, in final form 15 December 2008

Published 30 January 2009

Online at stacks.iop.org/JMM/19/035001

\begin{abstract}
A novel multi-frequency energy harvester has been designed and fabricated, which consists of three permanent magnets, three sets of two-layer copper coils and a supported beam of acrylic, while these coils are made of thin fire resistant 4 (FR4) substrates using a standard printed circuit board. The energy under the first, second and third resonant modes can be harvested, corresponding to the resonant frequencies of $369 \mathrm{~Hz}, 938 \mathrm{~Hz}$ and $1184 \mathrm{~Hz}$, respectively. The maximum output voltage and power of the first and second vibration modes are $1.38 \mathrm{mV}$, $0.6 \mu \mathrm{W}$ and $3.2 \mathrm{mV}, 3.2 \mu \mathrm{W}$ for a $14 \mu \mathrm{m}$ exciting vibration amplitude and a $0.4 \mathrm{~mm}$ gap between the magnet and coils, respectively. The feasibility study results are in good agreement with the theoretical calculations and show promising application potentials.
\end{abstract}

(Some figures in this article are in colour only in the electronic version)

\section{Introduction}

Recently a lot of interest has been attracted by MEMS energy harvesters capable of converting kinetic energy from ambient vibrations into electrical energy. Beeby et al [1] and Roundy et al [2] have reviewed three major energy transduction micromechanisms for harvesting energy from vibrations, namely, electromagnetic, piezoelectric and electrostatic approaches. Additionally, Arnold [3] has reviewed various micro-scale power generators based on the electromagnetic scheme. Most of the reported vibrationpowered harvesters provide the maximum output power when such devices operate at their mechanical resonances. However, in fact, the vibration frequency of environment excitation sources would vary from one case to another [2, 4]. For example, the vibration frequency of a desktop PC during normal operation is $543 \mathrm{~Hz}$, but the vibration frequency while running a $\mathrm{CD} \mathrm{ROM}$ is $154 \mathrm{~Hz}$ [5]. Therefore, it is necessary that the energy under different vibration frequencies with respect to a given MEMS harvester can be collected. The reported solutions for resolving this practical concern are categorized into two major groups. First of all, a wideband frequency approach has been demonstrated. Sari et al [6] have reported an array of cantilevers with different lengths and resonant frequencies. When this array of cantilevers is excited by a shaker across a band of vibration frequency, the electrical coils prepared on these cantilevers will generate current with the same frequency as the mechanical resonance of each cantilever. When the dimension and materials of cantilevers are designed and selected properly, $10 \mathrm{mV}$ voltage and $0.4 \mu \mathrm{W}$ power can be generated continuously within a frequency band of $800 \mathrm{~Hz}(4.2-5 \mathrm{kHz})$ from an array of 35 cantilevers. Besides, Roundy et al have reported a concept of making a multi-degree-of-freedom piezoelectric bimorph including multiple individual masses arranged along this bimorph beam for achieving a wider operation bandwidth of $17.5 \mathrm{~Hz}$ [7]. The second group is mainly working on multiple vibration modes, while each mode represents one resonant frequency. A piezoelectric multi-frequency energy converter is reported by Marco et al [8], which deployed a similar concept of multiple bimorph piezoelectric cantilevers with various masses at the cantilever end such that the 


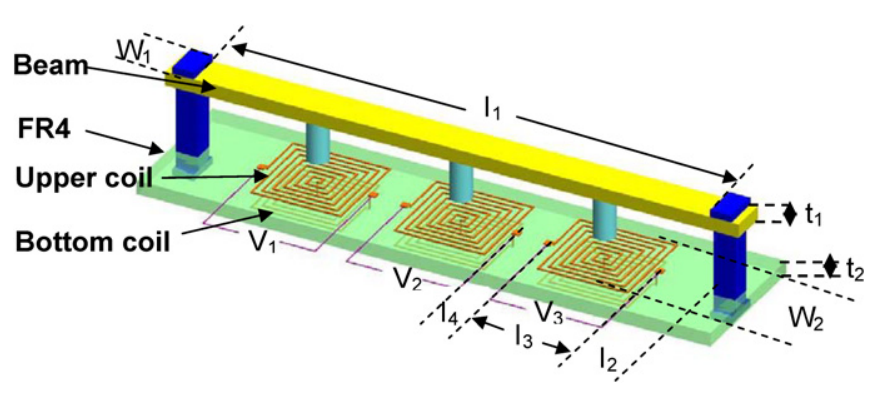

Figure 1. Schematic drawing of the FR4 energy harvester.

resulted resonant frequencies are different. In addition to the above-mentioned cantilever-array-based approaches, an $\mathrm{NdFeB}$ magnet assembled on a movable $\mathrm{Cu}$ plate which is supported by spiral shaped $\mathrm{Cu}$ springs has been reported by Neil et al [9].

On the other hand, most of the previously reported approaches are working on silicon-based solution. In view of the low-cost sensor network applications, it is very intriguing to explore polymer-based low-cost solutions. It is worthwhile mentioning that most commercially available magnets of high performance are typically in the volume of $10-50 \mathrm{~mm}^{3}$. Such a dimension is actually in line with the standard printed circuit board (PCB) technology. A PCB with multiple $\mathrm{Cu}$ coils could easily be fabricated in a very low cost way. In this feasibility study, we propose a simple polymer beam structure with multiple magnets, i.e., seismic masses, attached along the beam, while the beam can be vibrated on top of a stationary PCB substrate with multiple coils. The output from each individual coil is the function of the magnetic flux change attributed to the movable magnets on the said beam. It reveals a low-cost approach with the advantage of harvesting energy from vibrations of multiple frequencies.

\section{Design and modeling}

\subsection{Design of the device}

The schematic design of the vibration-based electromagnetic energy harvester based on three sets of coils and magnets is shown in figure 1 . The device includes three permanent magnets, three sets of bi-layer copper coil and a supported acrylic beam, while the coils are made of thin fire resistant 4 (FR4) substrates using standard PCB technology, and the acrylic beam is fabricated by laser machining. When the magnets vibrate and move toward the coils, the induced voltage will be generated from the coils according to Faraday's law of induction. The spiral layouts of coils on double sides of one FR4 substrate are made for allowing a current flow of two sides with opposite directions under the same magnetic flux direction. In other words, the compensation of generated current from two sides is avoided in this way.

\subsection{Mathematical modeling}

Inertial-based harvesters are essentially second-order, springmass systems. Assuming that an input displacement of $y(t)=$
$Y \sin (\omega t)$ was applied to the inertial frame, the relative velocity at the standard steady state can be expressed by equation (1) [10]:

$$
\dot{z}(t)=\frac{\left(\frac{\omega}{\omega_{n}}\right)^{2} \omega Y}{\sqrt{\left(1-\left(\frac{\omega}{\omega_{n}}\right)^{2}\right)^{2}+\left(2 \zeta_{m} \frac{\omega}{\omega_{n}}\right)^{2}}} \cos (\omega t+\varphi),
$$

where $\omega$ is the external vibration frequency, $Y$ is the amplitude of vibration, $\varphi$ is the phase angle, $\omega_{n}$ is the natural frequency of the system and $\xi_{m}$ is the mechanical damping ratio of the system.

For the fixed-fixed beams, the natural modes should be considered in the relative velocity equation. The relative velocity term in this case is given by the following equation [11]:

$$
\begin{aligned}
\dot{z}(t, x) & =\frac{\left(\frac{\omega}{\omega_{n}}\right)^{2} \omega Y \sin \frac{r \pi x}{L_{B}}}{\sqrt{\left(1-\left(\frac{\omega}{\omega_{n}}\right)^{2}\right)^{2}+\left(2 \zeta_{m} \frac{\omega}{\omega_{n}}\right)^{2}}} \cos (\omega t+\varphi), \\
r & =1,2,
\end{aligned}
$$

where $r$ is the $r$ th mode, $L_{B}$ is the length of the supported beam and $x$ is the location of the beam.

The induced open-circuit emf voltage $V_{\mathrm{em}}$ is given by Faraday's law [12]

$$
V_{\mathrm{em}}=-B L_{P} \dot{z}
$$

where $B$ is the magnetic field produced by the magnet and $L_{P}$ is the practical coil length.

Substituting equation (2) into (3),

$$
V_{\mathrm{em}}=-B L_{P} \frac{\left(\frac{\omega}{\omega_{n}}\right)^{2} \omega Y \sin \frac{r \pi x}{L_{B}}}{\sqrt{\left(1-\left(\frac{\omega}{\omega_{n}}\right)^{2}\right)^{2}+\left(2 \zeta_{m} \frac{\omega}{\omega_{n}}\right)^{2}}} \cos (\omega t+\varphi) .
$$

For the traditional large mass-/coil-type electromagnetic harvester, the instantaneous output power at steady state can be expressed by equation (5) [13]

$$
P(t)=\frac{1}{2} \frac{\left(B L_{P}\right)^{2} R_{L}}{\left(R_{L}+R_{C}\right)^{2}} \stackrel{\bullet}{z}^{2}
$$

where $R_{L}$ is the equivalent load resistance of the electricity circuitry and $R_{C}$ is the coil resistance.

Substituting equation (2) into (5),

$$
\begin{aligned}
P(t) & =\frac{1}{2} \frac{\left(B L_{P}\right)^{2} R_{L}}{\left(R_{L}+R_{C}\right)^{2}} \\
& \times \frac{\left(\frac{\omega}{\omega_{n}}\right)^{4} \omega^{2} Y^{2}\left(\sin \frac{r \pi x}{L_{B}}\right)^{2}}{\left(1-\left(\frac{\omega}{\omega_{n}}\right)^{2}\right)^{2}+\left(2 \zeta_{m} \frac{\omega}{\omega_{n}}\right)^{2}} \cos ^{2}(\omega t+\varphi) .
\end{aligned}
$$

From this expression, it can be derived that one condition leading to the maximum output value of $P(t)$ is $R_{L}=R_{C}$ when the device operates at the resonant frequency. In other words, when the loading resistor matches the internal resistance of the coil, the energy harvester can provide the maximum output power.

The efficiency of a harvester should be the ratio of the energy delivered to an electrical load to the input energy from 


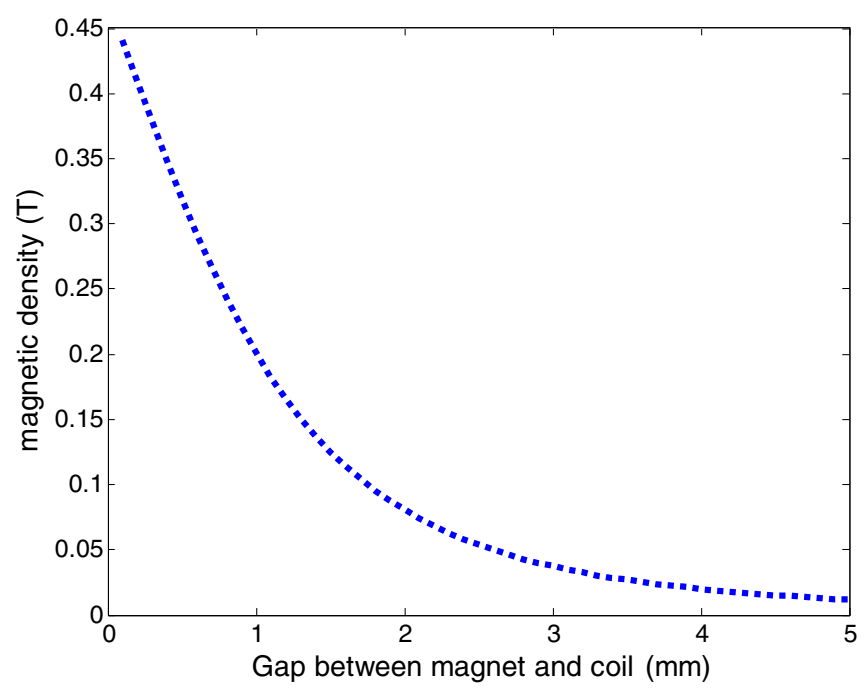

Figure 2. The magnetic density versus the gap of magnet and coils.

the excitation vibrations per cycle. Normally, the coupling coefficient, $k$, is a parameter related to the efficiency of the conversion from the external vibration energy to the energy stored within the harvester [14], which is the vector field on the $z$ axis. In the case of the electromagnetic harvester, the coupling coefficient is given by equation (7):

$$
\kappa^{2}=\frac{\left(B L_{P}\right)^{2}}{\kappa_{\mathrm{sp}} L},
$$

where $B$ is the magnetic flux density, $L_{P}$ is the total practical coil length, $k_{\mathrm{sp}}$ is the spring constant and $L$ is the inductance of the conductor.

The coupling coefficient depends on the structural design of the harvester. From equation (7), in order to improve the coupling coefficient, the flux density and the practical coil length need to be increased, while the spring remains constant, i.e. the stiffness of the supported beam should be decreased.

The maximum transmission coefficient is $\lambda_{\max }=$ $U_{\max } / U_{\text {in }}$ and is given by equation (8):

$$
\lambda_{\max }=\frac{\kappa^{2}}{4-2 \kappa^{2}},
$$

where $\lambda_{\max }$ is the maximum transmission coefficient, which is independent of loading conditions, $U_{\max }$ is the maximum output energy, $U_{\max }=\kappa_{\mathrm{sp}} \kappa^{2} z^{2} / 4, U_{\text {in }}$ is the total input energy, $U_{\text {in }}=\left(1-\kappa^{2} / 2\right) \kappa_{\mathrm{sp}} z^{2}$, which is from the excitation vibrations per cycle, and $z$ is the relative displacement of the conductor and magnetic field.

For a given cylinder magnet, the magnetic flux density $B$ is given by equation (9):

$$
B=\frac{B_{r}}{2}\left[\frac{(d+T)}{\left[R^{2}+(d+T)^{2}\right]^{1 / 2}}-\frac{d}{\left[R^{2}+d^{2}\right]^{1 / 2}}\right],
$$

where $d$ is the distance from the coil to the magnet, $B_{r}$ is the residual magnetic flux density, $R$ and $T$ are the radius and thickness of the magnet, respectively.

Figure 2 shows the relationship between the magnetic flux density and the gap of magnet and coils based on equation (9). It shows that the flux density clearly decreases with the increase
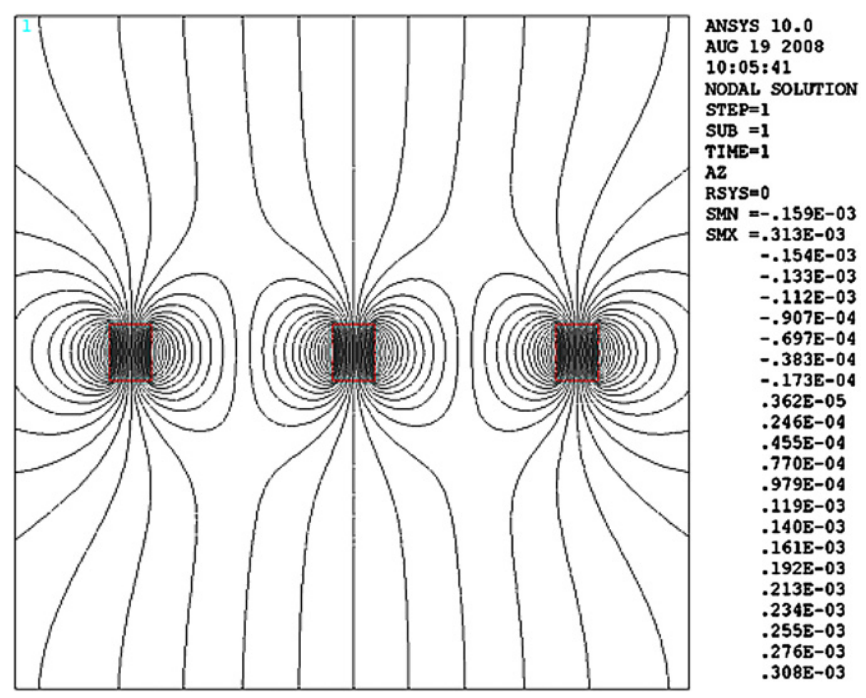

(a)
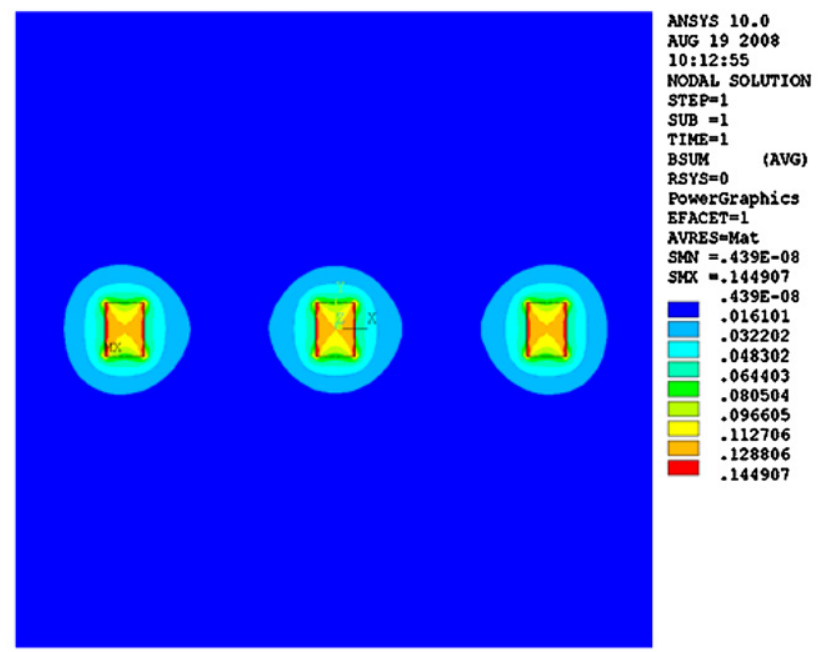

(b)

Figure 3. (a) 2D flux lines. (b) Flux densities distribution simulated by ANSYS.

in gap. Based on equations (4) and (6), the output voltage and power will decrease. A smaller gap between the magnet and coil is attributed to a higher output performance. In the mean time, it also increases the coupling coefficient. The dimension of the cylinder magnet is $3 \mathrm{~mm}$ in diameter and $4 \mathrm{~mm}$ in length. The properties of the magnet are shown in table 1. It is clear that the flux density will decrease sharply with increasing gap between the magnet and coils. In order to define the space distribution of three magnets, the simulation results of the flux lines and flux densities using the software of ANSYS are shown in figures 3(a) and $(b)$, respectively. There are few magnetic flux lines observed when the distance between magnets is above $1.6 \mathrm{~cm}$. Thus there is no interaction between each set of coil and magnet. Otherwise the output performance of each set will be affected by adjacent sets. Therefore the distance between two magnets is kept at $1.6 \mathrm{~cm}$ in our experiment. The designed structural parameters are listed in table 1 . 
This suspended beam with integrated three pieces of magnets exhibits multi-degree-of-freedom vibrations. Thus the vibration characteristics of the energy harvester have been studied by using the ANSYS finite element analysis. The material properties of the acrylic beam are shown in table 1 . Figure 4 gives the mode shapes of the first, second and third vibration modes corresponding to resonant frequencies of $346 \mathrm{~Hz}, 948 \mathrm{~Hz}$ and $1145 \mathrm{~Hz}$, respectively. When the vibration mode matches with the first resonant frequency, the middle magnet, i.e., the second magnet, will reach its maximum amplitude. The maximum vibration amplitude of the first and third magnets will be obtained at the second mode, i.e., the resonant frequency of $948 \mathrm{~Hz}$. Moreover, the third mode is a rotational mode and only the second coil can output the maximum voltage.

\section{Experimental results and discussion}

Figure 5 shows the fabricated prototype used for testing. The coils of FR4 are mounted on the acrylic, the magnets are glued onto the beam, and the cylinder of the acrylic is used for controlling the gap between the magnets and coils. Based on the simulation result of the flux density, the coil area is designed for $1 \mathrm{~cm} \times 1 \mathrm{~cm}$, and the distance between each coil is set at $1 \mathrm{~cm}$. For the standard PCB technology, the minimum coil line width is $254 \mu \mathrm{m}$, and each layer of coils consists of ten turns.

Figure 6 shows the experimental testing platform. This setup consists of an electromagnetic shaker (Gearing \& Watson Electronics Ltd V20, UK), a dynamic signal analyzer (Agilent 35670A), an accelerometer (ADI iSensor GS09001, USA), a power amplifier (Brüel \& Kjær WQ1108, Demark) and a computer to collect information through the GPIB port. The accelerometer is attached to the shaker in order to measure the input acceleration. The dynamic signal analyzer drives the shaker through an amplifier with an input voltage of sinusoidal signal $V_{\mathrm{i}}$ so as to control the amplitude and frequency of the shaker. The frequency response and output voltage from each individual pair of magnet and coil are also recorded by the different channels of the dynamic signal analyzer.

Figures $7(a)$ and $(b)$ show the variation in output voltage with the excitation frequency from 0 to $1500 \mathrm{~Hz}$ regarding to an initial gap of $1.1 \mathrm{~mm}$ between magnets and coils. As can be seen from figure 7(a), the resonant frequency of the first mode is about $369 \mathrm{~Hz}$ with the maximum output voltage $V_{2}$ being $0.088 \mathrm{mV}$ under the acceleration of $0.76 \mathrm{~g}(\mathrm{~g}=$ $9.8 \mathrm{~m} \mathrm{~s}^{-2}$ ). Meantime, $V_{1}$ and $V_{3}$ in this mode are $0.05 \mathrm{mV}$ and $0.06 \mathrm{mV}$, respectively, and the slightly different values are attributed to the fabrication deviation and limitation of the assembly accuracy, where $V_{2}$ represents the output voltage from the middle coil, and $V_{1}$ and $V_{3}$ mean the output voltages from left coil and right coil showed in figure 5 . When we put these three coils in serial connection, the accumulated output voltage is about $0.2 \mathrm{mV}$. Figure $7(b)$ shows the second and third vibration modes of the device at $938 \mathrm{~Hz}$ and $1184 \mathrm{~Hz}$, respectively. For the second mode, the output maximum voltage $V_{1}$ and $V_{3}$ are $0.009 \mathrm{mV}$, which are smaller than $V_{2}$ in the first mode. Under the same acceleration excitation, the

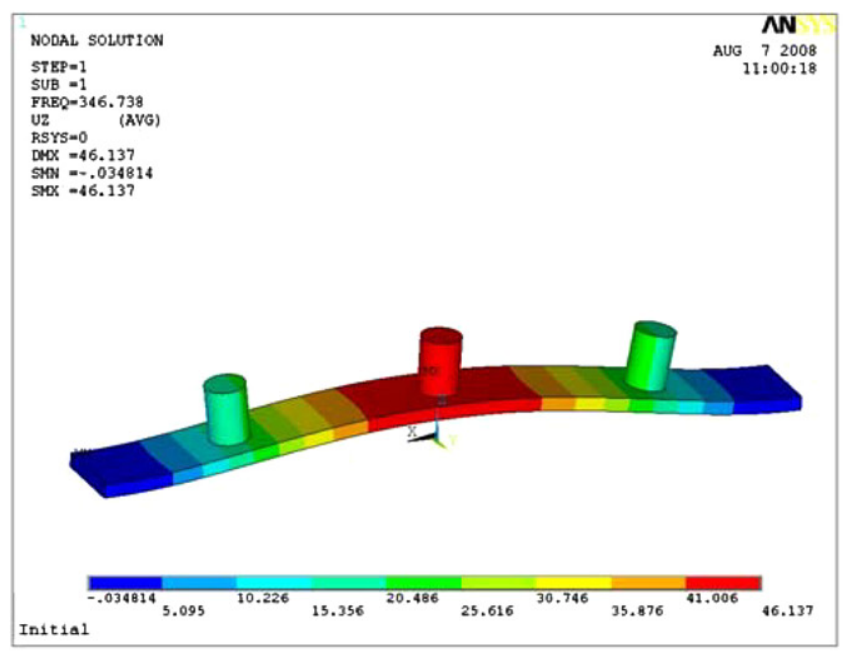

(a)

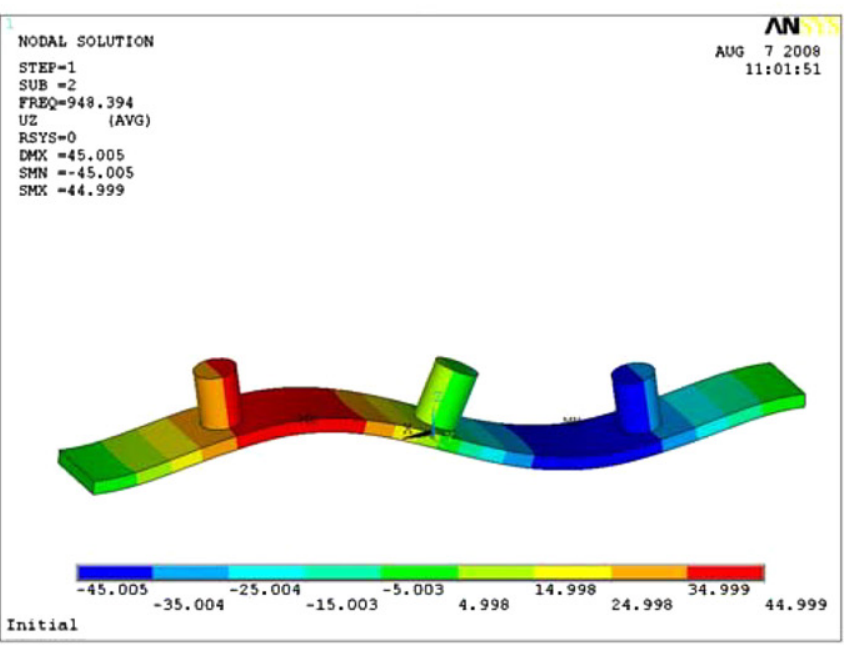

(b)

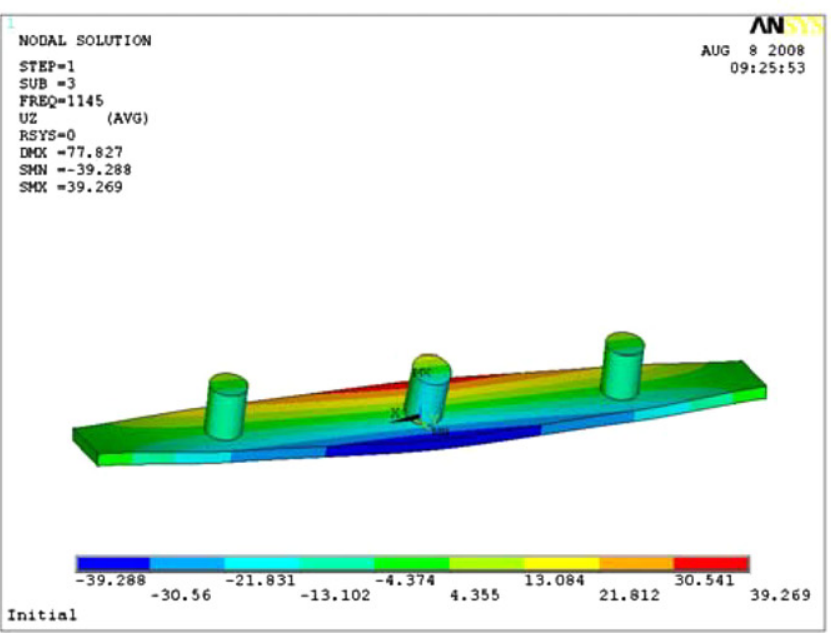

(c)

Figure 4. Finite element simulations for three different resonant vibration modes: (a) the natural frequency of the first mode vibration is $346 \mathrm{~Hz}$; $(b)$ the natural frequency of the second mode vibration is $948 \mathrm{~Hz}$; $(c)$ the natural frequency of the third mode vibration is $1145 \mathrm{~Hz}$.

vibration amplitude will decrease as the vibrated frequency increases, so the value of the maximum output voltage 
Table 1. Material properties of magnet and supported beam and structural parameters.

\begin{tabular}{llll}
\hline & Parameter & Description & Value \\
\hline Material properties & $E_{1}$ & Nd magnet Young's module & $41.4 \mathrm{e} 9 \mathrm{~Pa}$ \\
& $\rho_{1}$ & Nd magnet density & $7.4 \mathrm{e} 3 \mathrm{~kg} \mathrm{~m}$ \\
& $v_{1}$ & Nd magnet Poisson & 0.28 \\
& $E_{2}$ & Acrylic beam Young's module & $3.2 \mathrm{e} 9 \mathrm{~Pa}$ \\
& $\rho_{2}$ & Acrylic beam density & $1.4 \mathrm{e} 3 \mathrm{~kg} \mathrm{~m}^{3}$ \\
& $v_{2}$ & Acrylic beam Poisson & 0.40 \\
Structural parameters & $l_{1}$ & Length of the acrylic beam & $5.4 \mathrm{e}-2 \mathrm{~m}$ \\
& $W_{1}$ & Width of the acrylic beam & $6 \mathrm{e}-3 \mathrm{~m}$ \\
& $t_{1}$ & Thickness of the acrylic beam & $1 \mathrm{e}-3 \mathrm{~m}$ \\
& $l_{3}$ & Length of the single-layer coil area & $1 \mathrm{e}-2 \mathrm{~m}$ \\
& $W_{2}$ & Width of the single-layer coil area & $1 \mathrm{e}-2 \mathrm{~m}$ \\
& $t_{2}$ & Thickness of the coil substrate & $5 \mathrm{e}-4 \mathrm{~m}$ \\
& $l_{2}$ & Distance between coil and fixed end & $1.1 \mathrm{e}-2 \mathrm{~m}$ \\
& $l_{4}$ & Distance between coils & $1 \mathrm{e}-2 \mathrm{~m}$ \\
\hline
\end{tabular}

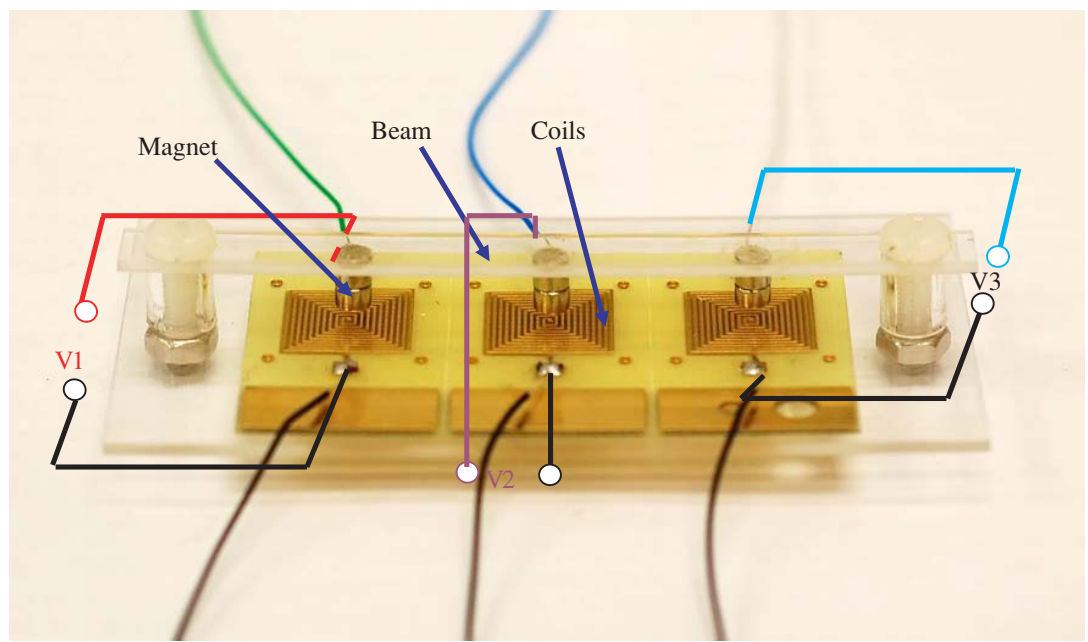

Figure 5. Photograph of the whole device.

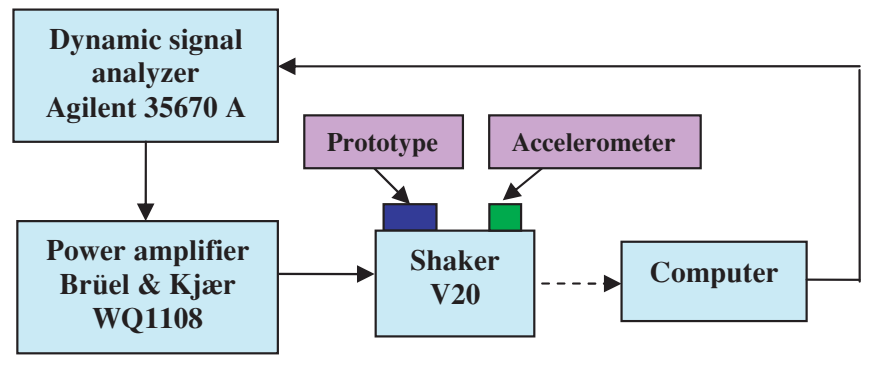

Figure 6. Block diagram of the test setup.

Table 2. The resonant frequency comparison between the simulated and experimental results.

\begin{tabular}{lrc}
\hline Vibration mode & Simulation & Experimental \\
\hline 1st mode & $346 \mathrm{~Hz}$ & $369 \mathrm{~Hz}$ \\
2nd mode & $948 \mathrm{~Hz}$ & $938 \mathrm{~Hz}$ \\
3rd mode & $1145 \mathrm{~Hz}$ & $1184 \mathrm{~Hz}$ \\
\hline
\end{tabular}

decreases in the second and third modes. The measured resonant frequencies are in good agreement with the simulation results as shown in table 2.
Figure 8 shows the output voltage versus various excitation amplitudes of the shaker derived from accelerometer output signals at the first resonant mode. In the case of $1.9 \mathrm{~g}$ acceleration, i.e. $3.5 \mu \mathrm{m}$ excitation amplitude, the output voltage $V_{2}$ is $0.231 \mathrm{mV}$. We observe that there is a linear relationship between the output voltage and the excitation vibration amplitude. But it is clearly seen that the slope of $V_{2}$ is larger than that of $V_{1}$ and $V_{3}$, which is attributed to a larger relative velocity of the middle magnet based on equation (2).

The magnetic flux density is a function of the gap distance between the magnets and coils as described in equation (7). Figure 9 shows the output voltage versus different initial gaps between magnet and coils under $1.4 \mu \mathrm{m}$ excitation amplitude $(0.76 \mathrm{~g})$. It is clear that the smaller gap contributes to the enlarged output voltages. From figures 8 and 9, it is obviously observed that there is a small discrepancy in the output $V_{2}$ voltage between simulated and experimental results, which is attributed to a larger damping ratio with a higher relative velocity of the middle magnet than $V_{1}$ and $V_{3}$ from equation (4). But there is a good agreement between the simulation and experimental results of $V_{1}$ and $V_{3}$.

Based on the modeling of the device, the practical coil length will affect the output voltage. Figure 10 shows the 


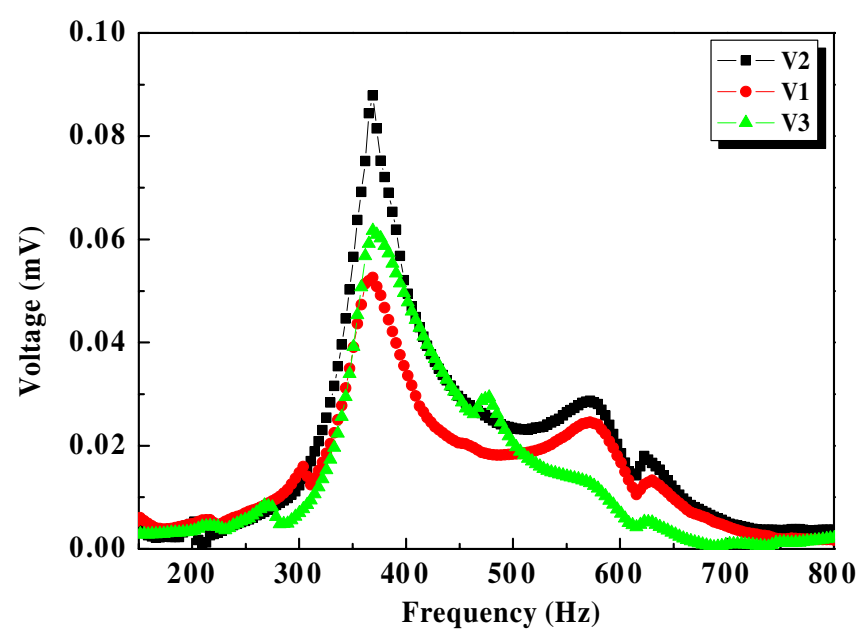

(a)

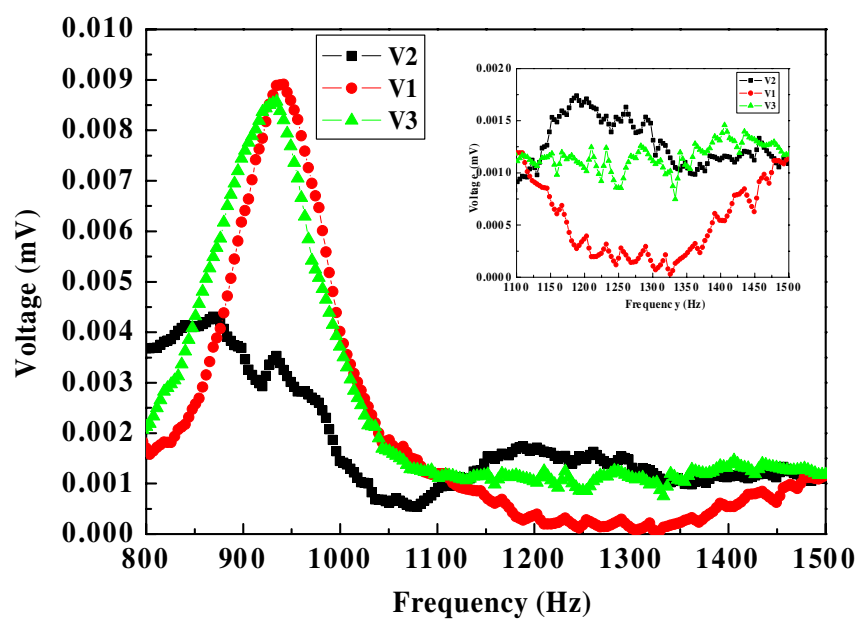

(b)

Figure 7. Open-circuit output voltage of the first, second and third vibration modes: $(a)$ the output voltage versus frequency from $150 \mathrm{~Hz}$ to $800 \mathrm{~Hz}$; $(b)$ the output voltage versus frequency from $800 \mathrm{~Hz}$ to $1500 \mathrm{~Hz}$.

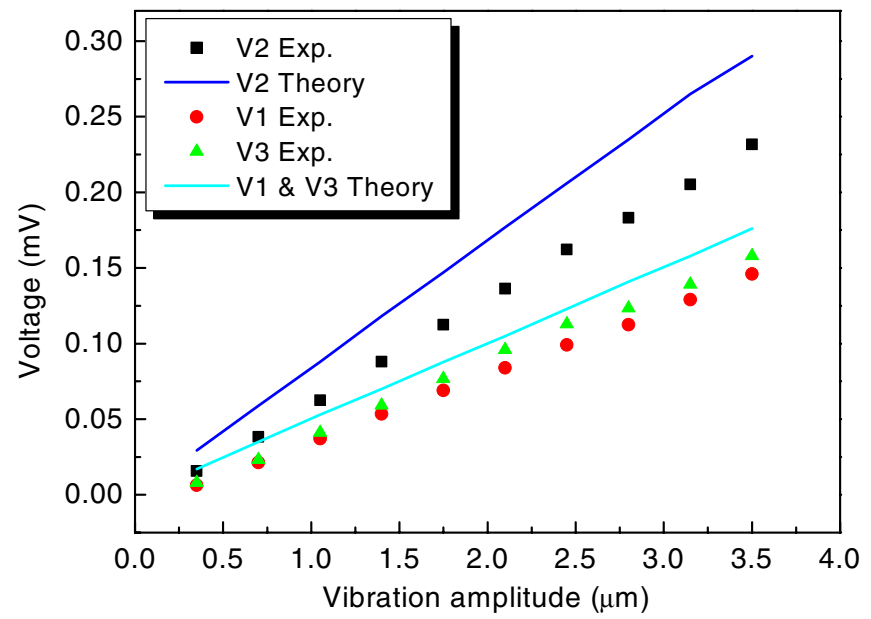

Figure 8. Voltage output versus vibration amplitude under the $1.1 \mathrm{~mm}$ initial gap.

output voltage from three sets of coils versus two kinds of coil substrate, i.e., two and four coil layers, where the excitation

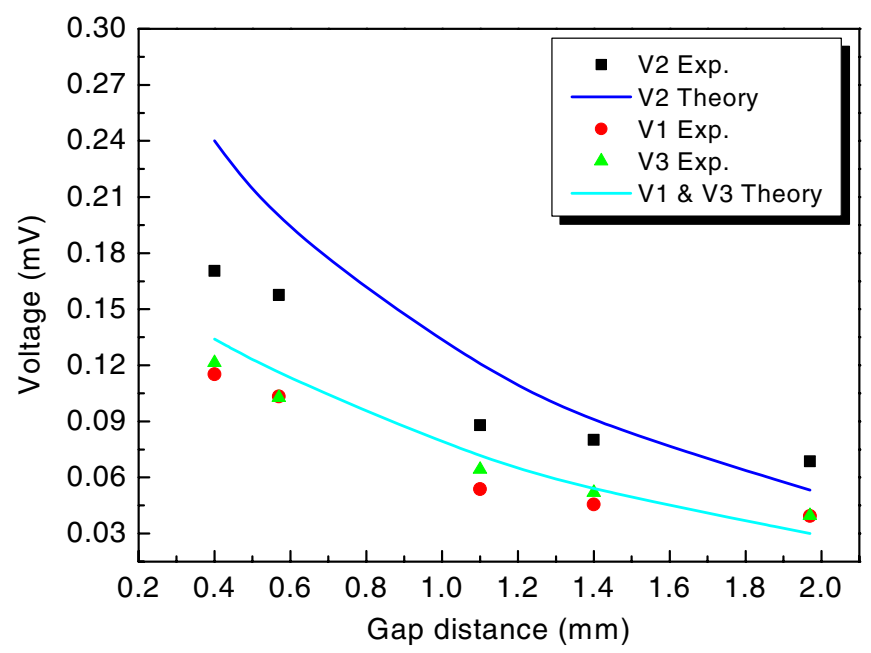

Figure 9. Voltage output versus gap distance under the $1.4 \mu \mathrm{m}$ excitation amplitude.

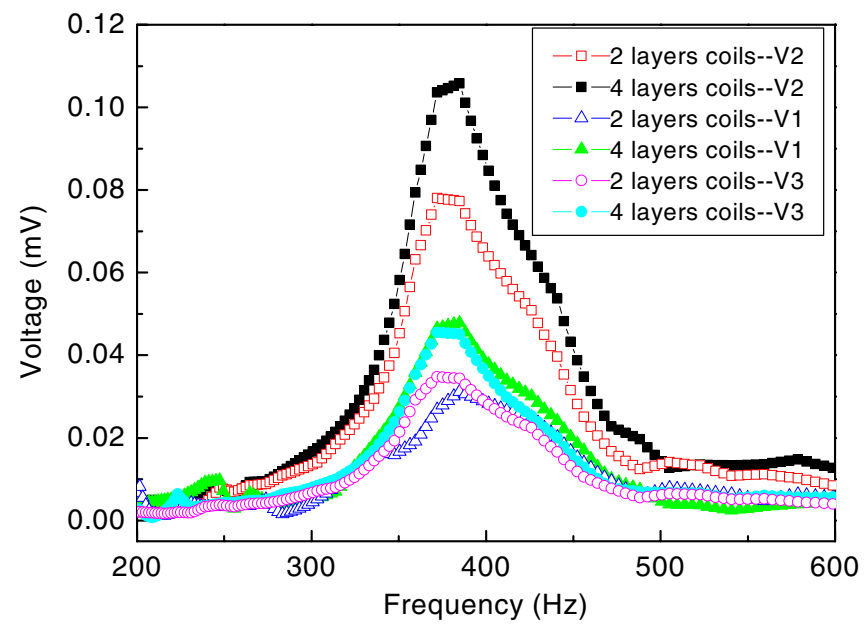

Figure 10. Voltage output versus vibration amplitude under $1.4 \mu \mathrm{m}$ excitation amplitude and $1.1 \mathrm{~mm}$ initial gap.

amplitude is $1.4 \mu \mathrm{m}$ and the initial gap between magnets and coils is the same as the one in the previous case, i.e., $1.1 \mathrm{~mm}$. It is seen that the voltage increases as the number of coil layers is increased. On the other hand, the increase of the length of magnets will increase the flux density and the output voltage of the device. Moreover, the resonant frequency of the energy harvester will easily decrease by using this method. Thus the actual resonant frequency can be tuned to each specific application.

The internal resistance of the twenty-turn coil for twolayer coils was $0.8 \Omega$. Figure 11 shows the output power versus various load resistances under the first mode vibration at $369 \mathrm{~Hz}$, where the excitation amplitude and initial gap between magnet and coils are $14 \mu \mathrm{m}$ and $0.4 \mathrm{~mm}$, respectively. When a matched resistor is $0.8 \Omega$, the maximum output power values of $0.6 \mu \mathrm{W}$ and $1.157 \mu \mathrm{W}$ are measured from the second coil $\left(V_{2}\right)$ and from all coils $\left(V_{\text {all }}\right)$ in serial connection. Regarding the same device configuration, the maximum output power of $3.2 \mu \mathrm{W}$ is measured from the first coil $\left(V_{1}\right)$ under the second mode vibration at $938 \mathrm{~Hz}$ as shown in figure 12 . It is 


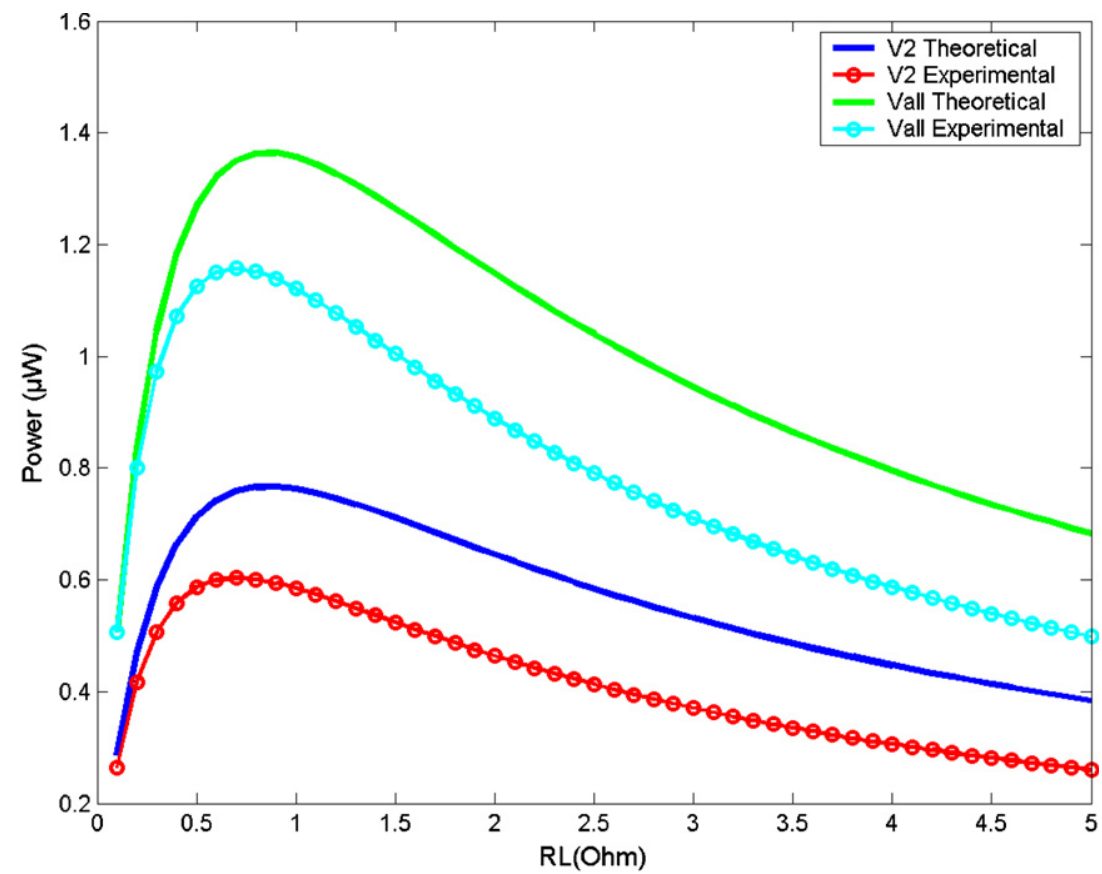

Figure 11. Output power versus load resistance measured at the first natural frequency, where the power of the second coil $\left(V_{2}\right)$ and all coils in serial connection $\left(V_{\text {all }}\right)$ are displayed.

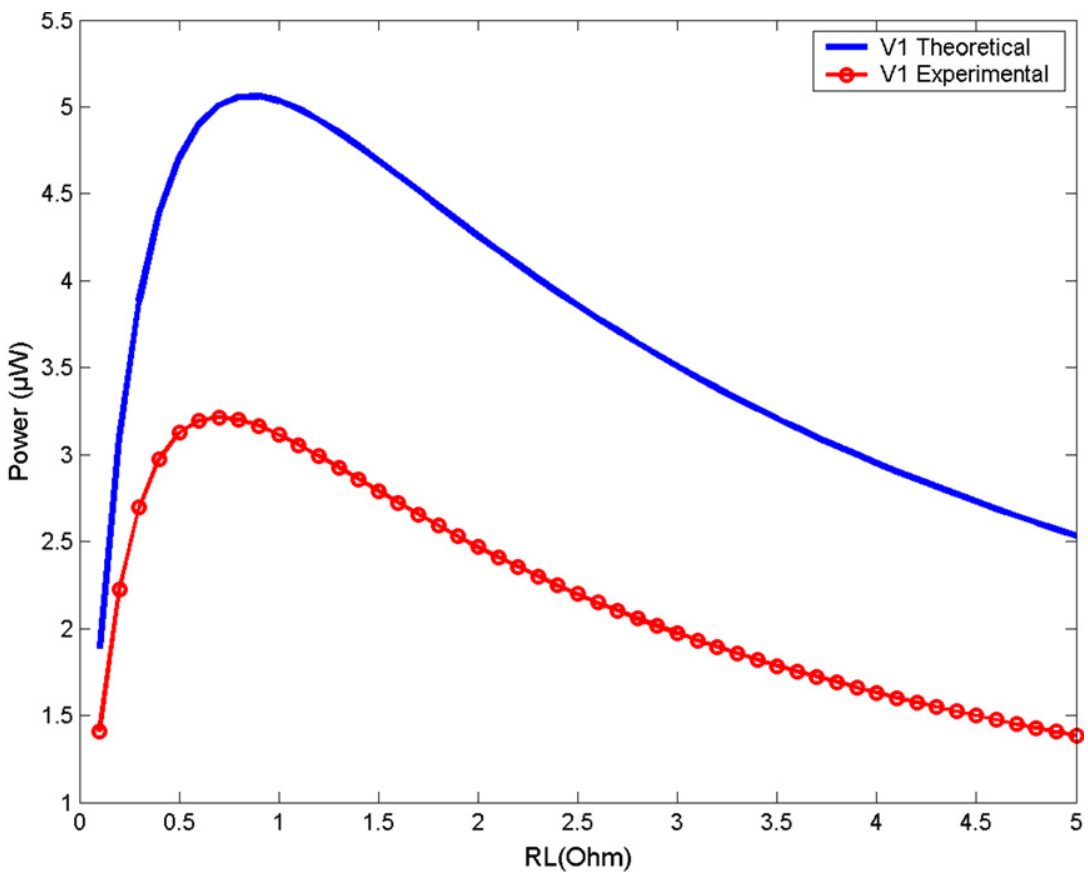

Figure 12. Output power versus load resistance measured at the second natural frequency, where the power of the first coil $\left(V_{1}\right)$ is displayed.

worthwhile to highlight that $V_{3}$ has the same value as $V_{1}$ with $180^{\circ}$ phase difference. Without having all coils in serial connection, we can separately collect current from each coil and store such energy in individual circuits. It is a way of allowing us to fully utilize the energy collected from the first and third coils. Additionally, the output power from the second coil is expected to be varied small due to the small vibration amplitude. Thus we do not discuss its effect. On the other hand, regarding the $14 \mu \mathrm{m}$ exciting vibration amplitude and the $0.4 \mathrm{~mm}$ gap between the magnet and coils, the maximum output voltage of $V_{2}$ at the first vibration mode and $V_{1}$ at the second vibration mode are measured as $1.38 \mathrm{mV}$ and $3.2 \mathrm{mV}$, respectively. As shown in figures 11 and 12, there is a discrepancy between the measured output power curves and calculated curves, which is mainly attributed to the existence of back emf when current passes through the coils. The effect 
is significant when the load resistance is very small because a larger current will pass through the coils [9].

\section{Conclusion}

In this paper, a novel multi-frequency energy-harvesting technique based on the electromagnetic method is proposed, designed and characterized. Optimization of the energyharvesting performance has been characterized in terms of several aspects: (a) increasing the exciting vibration amplitude; (b) increasing the number of coil turns and layers; (c) decreasing the gap between the magnet and coils; (d) increasing the magnetic field intensity. The experimental results show that the vibration energy under the three environment exciting frequencies of $369 \mathrm{~Hz}, 938 \mathrm{~Hz}$ and $1184 \mathrm{~Hz}$ can be harvested. In the case of $14 \mu \mathrm{m}$ exciting vibration amplitude and $0.4 \mathrm{~mm}$ gap between magnet and coils, the maximum output power of the first vibration mode is $0.6 \mu \mathrm{W}$ from the second magnet, while a total output power of $1.157 \mu \mathrm{W}$ can be obtained when the three sets of coils connect serially. Besides, the maximum output power of the second vibration mode is $3.2 \mu \mathrm{W}$ from the first coil with the same device configuration and testing setup. The major advantage of our device is low cost and capability of harvesting more energy from vibrations of multi-frequency.

\section{Acknowledgments}

Chengkuo Lee, PI of an A*STAR project entitled 'Si MEMS based energy harvesters for wireless medical sensor nodes', would like to thank A*STAR HOME 2015 National Research Programme (SERC grant number: 0621150043) for the funding of this project and in-kind contribution from Institute of Microelectronics, A*STAR.

\section{References}

[1] Beeby S P, Tudor M J and White N M 2006 Energy harvesting vibration sources for microsystems applications Meas. Sci. Technol. 17 175-95

[2] Roundry S, Wright P K and Rabaey J 2003 A study of low level vibrations as a power source for wireless sensor nodes Comput. Commun. 26 1131-44

[3] Arnold D P 2007 Review of microscale magnetic power generation IEEE Trans. Magn. 43 3940-51

[4] Mitcheson P D, Green T C, Yeat E M and Holmes H S 2004 Architectures for vibration-driven micropower generators J. Microelectromech. Syst. 13 429-40

[5] Swee L K, Neil M W and Harris Nick R 2008 A novel piezoelectric thick-film free-standing cantilever energy harvester Proc. Eurosensors XXII (Dresden, Germany) pp 395-9

[6] Sari I, Balkan T and Kulah H 2008 A electromagnetic micro power generator for wideband environmental vibrations Sensors Actuators A 145 405-13

[7] Roundry S et al 2005 Improving power output for vibrationbased energy scavengers IEEE Pervasive Comput. 4 28-36

[8] Marco F, Vittorio F, Michele G, Darioli M and Andrea T 2008 Piezoelectric multifrequency energy converter for power harvesting in autonomous microsystems Sensors Actuators A 142 329-35

[9] Neil N H, Wong H Y, Li W J, Leong H W and Wen Z Y 2002 A laser-micromachined multi-modal resonating power transducer for wireless sensing systems Sensors Actuators A 97-98 685-90

[10] Kelly S G 1997 Fundamentals of Mechanical Vibrations (New York: McGraw-Hill)

[11] Meirovitch L 2001 Fundamentals of Vibrations (New York: McGraw-Hill)

[12] Hayt W H 1981 Engineering Electromagnetics (New York: McGraw-Hill)

[13] Kulah H and Najafi K 2008 Energy scavenging from lowfrequency vibrations by using frequency up-conversion for wireless sensor applications IEEE Sensors J. 8 261-8

[14] Roundy S 2005 On effectiveness of vibration-based energy harvesting J. Intell. Mater. Syst. Struct. 16 809-23 